\title{
Exploring the Reproductive Health Needs of Violent Women: A Qualitative Study
}

\author{
Anvar Sadat Nayebi Nia ${ }^{\circledR}$, Mahrokh Dolatian $^{2^{\star}}$, Seyedeh Batool Hasanpoor-Azghady ${ }^{3}$, Abbas Ebadi $^{4}$, \\ Alirez Akbarzadeh-Baghban ${ }^{5}$
}

\begin{abstract}
Objectives: Violence against women is one of the most serious social problems in any cultures and communities, affecting the reproductive health of this gender. Accordingly, this study aimed to determine the reproductive health needs of violent women. Materials and Methods: This qualitative study was conducted on 19 domestically abused women and 9 key informants. The study population was selected through purposive sampling technique and the data were collected to the point of saturation using unstructured individual interviews, observation, and field notes. Eventually, data were analyzed by MAXQDA software using conventional content analysis.

Results: The main concepts obtained from the data were categorized into 5 major themes, including the need for motivating selfcare, empowering women against oppression, having a safe sex life, promoting the capacity of the reproductive health system in the support of domestically abused women, and training males to involve in reproductive health issues.

Conclusions: According to the results of the study, domestic violence is probably related to specific reproductive health needs among females at individual, family, and society levels. Therefore, it is essential to determine the needs of domestically abused women in order to improve their general and reproductive health.

Keywords: Reproductive health, Needs, Women, Domestic violence
\end{abstract}

\section{Introduction}

Over the past few years, violence against women has turned into one of the most serious social challenges in different cultures or societies (1). The World Health Organization (WHO) defines intimate partner violence as the behaviors leading to physical, sexual, and psychological damage. These behaviors mainly include physical and sexual violence, psychological abuse, and various controling attitudes (2). Generally, 35\% of women in the world experience physical or sexual violence imposed by their spouse (30\%) or non-sexual partner (3).

Abused women commonly experience physical, psychological, and health outcomes, as well as acute and chronic reproductive health issues (4). These issues can include general health and specific reproductive health problems such as low birth weight, preterm birth, pregnancy- and delivery-related problems, unwanted pregnancy, miscarriage, HIV, sexually transmitted infections $(3,5,6)$, and postpartum depression (7).

In a cross-sectional study conducted by Miller et al on 1278 females aged $16-29$ years, $53 \%$ of sexually- or physically-abused women reported spousal violence.
Furthermore, 9\% and 35\% of them had an experience of imposed pregnancy and spousal control over fertility, respectively (8). In addition, Ludermir et al investigated 1045 women in Brazil and observed a positive and significant relationship between psychological violence during pregnancy and postpartum depression (9).

Healthcare sector plays a significant role in responding to violence against women and providing these women with the required services such as sexual and reproductive healthcare needs is considered as one of the important measures that can be taken in this regard (3). It is important to identify the relationship between violence and reproductive health, especially due to the lack of adequate information on the reproductive health needs of the abused women. Despite the importance of this issue, previous studies have paid little attention to the various healthcare needs of women exposed to spousal violence. Culture exerts a significant effect on different aspects of this issue. As mentioned earlier, spousal violence is the most prevalent type of violence against women. However, no native study is available about the reproductive health needs of these women based on the 
experiences of domestically abused women and the key informants. Therefore, considering this background in mind, the present qualitative study sought to evaluate the reproductive health needs of violent women.

\section{Materials and Methods}

The current qualitative study was performed on domestically abused women and key informants at the healthcare and forensic centers of Tehran (Iran) employing conventional content analysis. In addition, a purposive sampling method was used to select the study population. Therefore, the interviews continued until the point of saturation, which led to the selection of 18 domestically abused women and nine experts in the field. The inclusion criteria for the abused women were domestic violence approval as determined by the WHO questionnaire, as well as the lack of any observable physical or psychological diseases, drug abuse, AIDS, and hepatitis, and finally, the age range of 20-49 years.

Furthermore, regarding the key informants, being knowledgeable about the subject of interest and showing a willingness to share opinions in this respect were considered as the inclusion criteria. It should be noted that the sampling was conducted with maximum diversity in terms of age, the level of education, socioeconomic status, and the number of children.

Data were collected employing non-structured individual interviews, observation, and field notes. During the interviews, the non-verbal behaviors of the subjects were taken into account as well. For the domestically abused women, the interview was started with an open question (i.e., "Could you please talk about the events occurred between you and your spouse and the unjustified behaviors of your spouse?"). Depending on the following conversations, the second question was raised (i.e., "Under this circumstance, what conditions or facilities could help you?" The interview was continued with questions covering the reproductive health needs of the subjects. The main questions for the interview were developed and edited by our research team and referees.

The interview sessions lasted for 35-75 minutes. On the other hand, the interview with the key informants was launched with an open question about the reproductive health needs of the domestically abused women and then followed by asking exploratory questions. By considering the time, discussions continued until the point that the interviewer ensured the thorough comprehension of the presented concepts. These interview sessions lasted about 20-50 minutes.

At the end of each interview and upon the interviewees' consent, the recorded data were transcribed verbatim after 2-3 times of listening, and eventually, typed. The obtained data were analyzed simultaneously with the data collection process through conventional content analysis. To this end, the interview transcriptions were reviewed several times in order to obtain a complete conceptualization of the content. Subsequently, some codes were extracted based on the semantic units derived from the women' descriptions and categorized according to their differences or similarities to determine the intended themes. Finally, data analysis was performed in MAXQDA, version 10 (VERBI, Germany).

Various methods were applied to ensure the credibility of the data based on Guba and Lincoln's proposed criteria as follows.

- Taking into account diversity in the subjects;

- Having long contact with the subjects and research setting;

- Constantly evaluating the data;

- Recording the women' voices;

- Immediately transcribing and analyzing the data following the interviews;

- $\quad$ Preparing feedback for the subsequent interviews.

Moreover, the data were assessed and approved by external observers and some of the study subjects. Similarly, the transferability of the data was ensured by providing information for some of the abused women who were not among the participants of the study, and asking them to compare the results with their own experiences.

\section{Results}

The results were obtained through the analysis of the collected information by interviewing 18 domestically abused women within the age range of 23-45 years and 9 key informants (Table 1). The mean work experience of the subjects was 20.6 years. Regarding the education level of the key informants, eight participants had $\mathrm{PhD}$ and one case had a master degree with the specialties in law (judgment), forensics, obstetrics and gynecology, reproductive health, psychology, sexual therapy, sociology, and general health management.

The concepts obtained from the interviews, including 5 main themes and their subcategories, are presented in Table 2 .

\section{Need to Motivate Self-care}

Need to Improve the Sense of Self-efficacy in Health

The creation of sensitivity toward the risk of sexually transmitted diseases is one of the main pillars of selfefficacy in reproductive health. On the other hand, the lack of attention to risky sexual behaviors is one of the factors that should be considered as well. In this regard, one of the women (A 40-year-old female with academic education) declared that "Finally, I found that he had sexual relations with others. The doctor had warned me about his suspicious relationships, but I paid no attention."

Sensitivity toward safe maternal behaviors is a matter of fundamental importance regarding self-efficacy in reproductive health. The timely implementation of pregnancy care, as one of the safe maternal behaviors, is neglected by the majority of the domestically abused women. This was clearly indicated by a 38 -year-old female 
Table 1. Demographic Characteristics and Domestic Violence Conditions of the Participants

\begin{tabular}{|c|c|c|c|c|c|c|}
\hline \multirow{2}{*}{ Age } & \multirow{2}{*}{ Education } & \multirow{2}{*}{ Duration of Marriage (y) } & \multirow{2}{*}{ Number of Children } & \multicolumn{3}{|c|}{ Domestic Violence Condition } \\
\hline & & & & Physical & Sexual & Psychological \\
\hline 33 & Senior high school & 4 & 0 & Moderate & Severe & Severe \\
\hline 26 & Academic & 2 & 0 & Mild & Mild & Severe \\
\hline 32 & Elementary & 15 & 4 & Severe & No & Severe \\
\hline 42 & Junior high school & 27 & 2 & Moderate & Severe & Severe \\
\hline 38 & Academic & 14 & 1 & Mild & Moderate & Severe \\
\hline 39 & Junior high school & 19 & 1 & Severe & Severe & Severe \\
\hline 40 & Academic & 25 & 2 & Severe & Severe & Severe \\
\hline 37 & Senior high school & 13 & 1 & Moderate & Severe & Severe \\
\hline 28 & Senior high school & 4 & 0 & Severe & Severe & Severe \\
\hline 44 & Elementary & 23 & 2 & Severe & Severe & Severe \\
\hline 32 & Academic & 12 & 1 & Moderate & Moderate & Severe \\
\hline 25 & Elementary & 7 & 2 & Severe & Severe & Severe \\
\hline 45 & Junior high school & 28 & 3 & Severe & Severe & Severe \\
\hline 34 & Academic & 2 & 0 & Severe & Moderate & Severe \\
\hline 37 & Academic & 10 & 1 & Moderate & Severe & Severe \\
\hline 36 & Academic & 14 & 1 & Severe & Severe & Severe \\
\hline 35 & Senior high school & 18 & 2 & Severe & Severe & Severe \\
\hline 31 & Junior high school & 11 & 3 & Severe & Severe & Severe \\
\hline
\end{tabular}

Table 2. Main Themes and Their Subcategories

\begin{tabular}{|c|c|}
\hline Main Themes & Categories \\
\hline \multirow{2}{*}{ Need to motivate self-care } & Need to improve the sense of self-efficacy in health \\
\hline & Need to accept responsibility toward health \\
\hline \multirow[t]{2}{*}{ Need to empower women against violence } & Need to optimally design formal and informal support services \\
\hline & Need to improve compliance with violence \\
\hline \multirow{2}{*}{ Need to have a safe sex life } & Need to recognize the female role in sexual intercourse \\
\hline & Need to build a culture to improve the role of women in sexual relations \\
\hline \multirow{2}{*}{$\begin{array}{l}\text { Need to improve the capacity of the reproductive health } \\
\text { system in support of domestically abused women }\end{array}$} & Need to simple access to economic maternity and safe motherhood services \\
\hline & $\begin{array}{l}\text { Need to have accountable healthcare system to provide abused women with } \\
\text { healthcare services }\end{array}$ \\
\hline \multirow{2}{*}{ Need to train men to involve in reproductive health issues } & Need to improve the attitude and knowledge of men about reproductive health \\
\hline & Need for men's emotional investment during pregnancy \\
\hline
\end{tabular}

with senior high school education as she declared "I accept that I paid little attention to timely referral for receiving the necessary care. I was really depressed and not in the mood."

\section{Need to Accept Responsibility Toward Health}

Adherence to family planning is one of the responsibilities related to reproductive health. In this respect, one of the abused women (A 45-year-old female with senior high school education) complained that "I used to receive an injection, but they advised me not to use it during breastfeeding. I accepted their suggestion and then realized that I was pregnant."

The acceptance of responsibility for health requires believing in health behaviors. For instance, the identification of self-administered treatment outcomes as a non-healthy behavior is significantly essential. For instance, a 28-year-old female with high school diploma acknowledged that "I bought medication from pharmacies for my psychological problems without referring to any psychologist."

2- Need to Empower Women Against Violence

Need to Optimally Design Formal and Informal Support Services

The government can provide support services for empowering females through delivering the required services and enhancing the women's awareness of their marital and personal rights. In this respect, one of the key informants (PhD in Law, having 18 years of experience) argued that "Unfortunately, women have inadequate knowledge about their personal rights or their right in sexual relations. Therefore, their knowledge must be increased through the media or even educational classes held before marriage." 
Likewise, providing social security for the divorced and abused women is regarded as another prerequisite for a support service system, which may include the prevention of isolation and negative social effects. This issue was evidently raised by a key informant ( $\mathrm{PhD}$ in General Health, having 26 years of experience):

"Sometimes, an abused woman might be even rejected by society, which could result in negative social impacts, including addiction and prostitution, which must be prevented."

\section{Need to Improve Compliance With Violence}

Flourishing abused women' self-belief is considered as one of the required empowerments for them to comply with violence due to their damaged self-esteem. Accordingly, one of the key informants ( $\mathrm{PhD}$ in Psychology, having 20 years of experience) indicated that "Humiliation is one of the most important factors in violence, which reduces selfesteem."

Moreover, search for help should be strengthened in abused women. Tolerating and hiding the abusing behaviors of spouses is among the barriers against searching for help due to the fear of bad reputation or emotional attachment to the spouse. In this regard, a 39-year-old female with junior high school degree expressed that "First, I talked with no one about my problems so that not to taint my reputation. Therefore, I tolerated the abuses so that other people do not question my duties as a wife."

\section{Need to Have Safe Sex Life}

Need to Recognize the Female Role in Sexual Intercourse The males' negligence towards their spouses' sexual needs affects the sexual life of the females, one of the manifestations of which is the suppressed sexual desire due to the lack of peace with the spouse. In this respect, a 40-year-old woman (Having academic education) with tearful eyes stated that "Everyone asks me about my lack of sexual desire I have experienced during the past four years. My sexual needs were suppressed since I had no peace in this relationship."

The recognition of the role of woman in sexual relations requires institutional modifications in the sex area. For instance, it seems essential to adjust the family court rules on sexual relations of the couples. This issue was clearly indicated by a forensic specialist with 23 years of experience:

"There is no rule regarding the sexual intercourse that the woman has no desire for. There are multiple of such rules that need modification in our legislation system."

\footnotetext{
Need to Build Culture to Improve the Role of Women in Sexual Relations

Culture building in terms of sexual relations could include changing the traditional attitude toward sexual roles and dealing with inaccurate sexual beliefs. Scorning the sexual needs of the spouse has roots in inaccurate sexual beliefs.
}

In this respect, one of the females (A 42-year-old woman, with junior high school education) complained that " $\mathrm{He}$ laughed when I talked about my sexual needs. They were funny for him."

Adherence to traditional beliefs and the preference of the sexual needs of the spouse is a type of traditional attitude toward sexual roles which requires modification. One of these traditional beliefs was pinpointed by a 23 -year-old woman with senior high school education, as follows.

"I was told to be at the service of my husband in bed and I did the same thing."

4. Need to Improve the Capacity of Reproductive Health System in Support of Domestically Abused Women

Need to Simple Access to Economic Maternity and Safe Motherhood Services

The facilitation of access to maternity services is among the needs of the abused women. In general, these women are more prone to some diseases, including vaginal infections, irregular menstruation, and hormonal disorders.

"I experienced early menopause at this age." (A 39-yearold woman with junior high school education)

Given the increased negative outcomes of pregnancy in abused women (e.g., stillbirth), it is essential to provide these women with safe motherhood services. A 32-yearold woman with elementary education reported such a negative outcome:

"My child weighted $2.5 \mathrm{~kg}$ but born dead. I am not sure about the cause, maybe because he kicked me in the stomach to wake me up."

Need to Have Accountable Healthcare System to Provide Abused Women With Healthcare Services

The presence of experienced personnel in different sections of the healthcare system is essential for recognizing the abused women's needs and providing the required services. Furthermore, obtaining the trust of these women by the healthcare personnel is an important issue. A key informant (PhD in Public Health having 26 years of experience) expressed that "The first thing to do is to create trust in healthcare centers so that the abused women could freely talk about their problems."

Another important issue is the management of the service costs. A 44-year-old woman (With elementary education) apparently indicated to this point, as follows:

"He does support me financially. You know how high the expenses of doctors and tests are. That is why I am often forced to self-administer treatment for myself and my children."

\section{Need to Train Men to Involve in Reproductive Health Issues}

Need to Improve the Attitude and Knowledge of Men About Reproductive Health

The lack of males' cooperation regarding treating their spouse's sexually transmitted diseases is due to their 
inadequate knowledge in this regard. One of the females (A 39-year-old woman having junior high school education) argued that "Doctor prescribed medications for both of us due to my vaginal discharge, but he threw his medicine out and said it was ridiculous and that he would not take medicine for my disease."

Another issue is the spousal lack of participation in the prevention of pregnancy, which puts the life of the women at risk due to unwanted pregnancy. A 40-year-old woman with academic education indicated that "He did not use condoms and wanted to be comfortable. He mentioned that "If you get pregnant, you should abort it yourself."

\section{Need for Men's Emotional Investment During Pregnancy}

The lack of spousal support during pregnancy and postpartum period was another complaint of the women. This need was clearly indicated in the statement of a 36-year-old woman (With academic education) "I felt empty after delivery, I mean, any woman expects to receive more affection from her husband at the postpartum period, but I was deprived of such affection."

In addition, the continuation of spousal violence during pregnancy and postpartum period demonstrates the significance of male emotional investment during these sensitive periods. In this regard, a 25-year-old woman (With elementary education) complained that "During my first pregnancy, he whipped me so hard that blood clots appeared on my body."

\section{Discussion}

Based on the results of the current qualitative study and based on the experiences of domestically abused women and key informants, the reproductive health needs of the domestically abused women included the need to:

- motivate self-care;

- empower women against aggression;

- have a safe sex life;

- improve the capacity of the reproductive health system regarding supporting the abused women;

- train men to involve in reproductive health issues.

Self-care is identified as conscious actions and measures taken by individuals, families, and societies to promote health status (10).

In the current study, one of the methods to achieve selfcare was the enhancement of the sense of self-efficacy and accountability about health issues. In a study conducted by Weng et al, self-care behaviors were significantly higher in patients with higher levels of self-efficacy (11). Moreover, in a model designed by Pender for health promotion, selfefficacy and accountability toward health were introduced as the health components, which is in line with our findings (12).

Similarly, empowerment is a multi-dimensional and active process, which enables women to recognize their identity and ability concerning all aspects of life (13). According to the results of the present study, a need for women empowerment against violence included the need for providing official and non-official support services and improving compliance in response to violence. In this regard, one of the official support services is the government commitment to provide the necessary services.

According to the $\mathrm{WHO}$, one of the factors assisting the process of women empowerment is the government support for overcoming the current taboos and norms, acting as barriers to women's health (14). In a review study conducted by Murphy and Ting, implementing programs regarding how to cope with violence and search for help was regarded as one of the effective interventions against violence (15).

Similarly, Zakar et al introduced some of the methods of compliance with violence as searching for family support and accessible resources, receiving support via social networks while not having contact with the spouse (16). Likewise, in the current study, search for help and effective communication with the spouse were presented as the prerequisites for compliance with violence.

According to the definition provided by the WHO, a safe and satisfactory sex life is one of the components of sexual rights (17). In the present study, a need for having a safe sex life was identified as one of the essential requirements in the area of reproductive and sexual health. In addition, the results of studies by Nayebinia et al (18) and Razeghi et al (19) indicated that impaired sexual health was one of the consequences of spousal violence against women.

Further, Rashidi et al concluded that the level of knowledge, as well as cultural, social, and religious values were among the components of sexual health definition (20). The results of the above-mentioned study are in congruence with our findings regarding the need for changing traditional attitudes toward sexual roles and dealing with inaccurate sexual beliefs in order to have a safe sex life. Based on the results of the current study, the necessity of having easy access to economically-justified maternity services is fulfilled by the responsiveness of the healthcare system through providing proper services for the abused women. Furthermore, based on the results of Mohammed et al (21) and Rahman et al (22), intimate partner's violence was associated with inadequate prenatal care and the lower likelihood of assisted deliveries from the skilled provider.

However, this responsiveness requires the management of service costs and experienced personnel. In this regard, our findings are consistent with the results obtained by Masterson et al in Lebanon. They reported cost, distance or transportation, fear of unsuitable treatment, the lack of access to female doctors, and inappropriate provision of services as some of the barriers to access to reproductive healthcare (23). In a study conducted by Dolatian et al the lack of adequate education was reported as one of the barriers of care providers for monitoring domestic 
violence in women (24).

Moreover, in qualitative research performed by Bacchus et al the attitude and responsiveness of the healthcare professionals were the most important factors for discussing spousal violence by the women (25). In the present research, some of the needs related to training the men for involvement in reproductive health issues included the need for the improvement of their knowledge and attitude toward this issue and more emotional investment during pregnancy. Additionally, the results of a review study by Yargawa et al indicated that men's participation in prenatal care was associated with a lower likelihood of postpartum depression and more access to maternity healthcare (26).

In addition, Mullany conducted qualitative research on a number of couples in Nepal and indicated inadequate knowledge, social stigmas, and shame as some of the most important barriers to the men's participation in maternal and neonatal healthcare issues (27). Additionally, Kululanga et al reported sexual norms and healthcare system outcomes such as the female nature of maternal services were regarded as two major barriers to the participation of men in maternal and neonatal healthcare issues (28). In this regard, our findings are in line with the results of the aforementioned study.

\section{Conclusions}

In general, domestic violence can affect women's reproductive health needs. Some of the most important health problems of domestically abused women included the lack of sensitivity toward health, increased high-risk behaviors and low-quality life skills, insufficient family and social support, the lack of spousal emotional investment in various aspects of women's sexual, physical, and psychological health, along with the non-responsiveness of reproductive healthcare system.

Likewise, such problems would result in specific reproductive health needs at individual, familial, and social levels. With regard to the extracted needs in the present research, further studies are recommended to design and implement educational programs for the abused women and their spouses. Finally, it is suggested to conduct broader studies in order to investigate the generalizability of the results.

\section{Limitations}

In the current study, access to individuals who were eager to participate in the research was difficult due to the sensitivity of the topic under investigation.

\section{Conflict of Interests}

Authors declare that they have no conflict of interests.

\section{Ethical Issues}

This study was extracted from a doctoral dissertation on reproductive health in Shahid Beheshti University of
Medical Sciences (Tehran, Iran) with the ethical code of SBMU2.REC.1394.113 on September 29, 2015. In the present study, participants allowed us to use the tape recorder.

\section{Financial Support}

This research was supported by Shahid Beheshti University of Medical Sciences, Tehran, Iran.

\section{Acknowledgments}

Hereby, we extend our gratitude to all the participants, healthcare officials, and forensic center authorities of Tehran for their cooperation in sampling. In addition, we would like to thank the efforts made by Shahid Beheshti University of Medical Sciences for approving the research project.

\section{References}

1. Afkari ME, Latifi M, Taghdisi MH, Azam K, Estebsari F. Indicators of empowerment in women supported by Imam Khomeini relief committee of Gorgan in confrontation with violence. Journal of Payavard Salamat. 2013;7(1):32-41.

2. Krug EG, Mercy JA, Dahlberg LL, Zwi AB. The world report on violence and health. Lancet. 2002;360(9339):1083-1088. doi:10.1016/s0140-6736(02)11133-0

3. Garcia-Moreno C, Pallitto C, Devries K, Stockl H, Watts C, Abrahams N. Global and regional estimates of violence against women: prevalence and health effects of intimate partner violence and non-partner sexual violence. Geneva, Switzerland: World Health Organization; 2013.

4. de Sousa J, Burgess W, Fanslow J. Intimate partner violence and women's reproductive health. Obstet Gynaecol Reprod Med. 2014;24(7):195-203. doi:10.1016/j.ogrm.2014.04.012

5. Kishor S, Johnson K. Reproductive health and domestic violence: are the poorest women uniquely disadvantaged? Demography. 2006;43(2):293-307. doi:10.1353/ dem.2006.0014

6. Sudha S, Morrison S. Marital violence and women's reproductive health care in Uttar Pradesh, India. Womens Health Issues. 2011;21(3):214-221. doi:10.1016/j. whi.2011.01.004

7. Dolatian M, Hesami K, Shams J, Alavi Majd H. Relationship between violence during pregnancy and postpartum depression. Iran Red Crescent Med J. 2010;12(4):377-383.

8. Miller E, Decker MR, Raj A, Reed E, Marable D, Silverman JG. Intimate partner violence and health care-seeking patterns among female users of urban adolescent clinics. Matern Child Health J. 2010;14(6):910-917. doi:10.1007/ s10995-009-0520-Z

9. Ludermir AB, Lewis G, Valongueiro SA, de Araujo TV, Araya R. Violence against women by their intimate partner during pregnancy and postnatal depression: a prospective cohort study. Lancet. 2010;376(9744):903-910. doi:10.1016/ s0140-6736(10)60887-2

10. World Health Organization (WHO). Self-care in the context of primary health care. WHO; 2009.

11. Weng LC, Dai YT, Huang HL, Chiang YJ. Self-efficacy, self-care behaviours and quality of life of kidney transplant recipients. J Adv Nurs. 2010;66(4):828-838. doi:10.1111/ 
j.1365-2648.2009.05243.x

12. Pender NJ. Health promotion model manual. University of Michigan; 2011.

13. Janghorban R, Taghipour A, Latifnejad Roudsari R, Abbasi $\mathrm{M}$. Women's empowerment in Iran: a review based on the related legislations. Glob J Health Sci. 2014;6(4):226-235. doi:10.5539/gjhs.v6n4p226

14. World Health Organization (WHO). Women's empowerment and gender equality: Essential goals for saving women's lives. WHO; 2008. https://www.who.int/ gender-equity-rights/knowledge/who_fch_gwh_082/en/.

15. Murphy CM, Ting LA. Interventions for perpetrators of intimate partner violence: A review of efficacy research and recent trends. Partner Abuse. 2012;1(1):26-44. doi:10.1891/1946-6560.1.1.26

16. Zakar R, Zakar MZ, Kramer A. Voices of strength and struggle: women's coping strategies against spousal violence in Pakistan. J Interpers Violence. 2012;27(16):3268-3298. doi:10.1177/0886260512441257

17. World Health Organization (WHO). Defining sexual health: report of a technical consultation on sexual health, 28-31 January 2002, Geneva. Geneva: WHO; 2006.

18. Nayebinia AS, Dolatian M, Hasanpour Azghadi B, Ebadi A, Akbarzadeh Baghban A. Domestic violence and its association with domains of reproductive health in women: A systematic review. Journal of Mazandaran University of Medical Sciences. 2018;27(158):205-217.

19. Razaghi N, Parvizy S, Ramezani M, Tabatabaei Nejad SM. The consequences of violence against women in the family: a qualitative study. The Iranian Journal of Obstetrics, Gynecology and Infertility. 2013;16(44):11-20.

20. Hosein Rashidi B, Kiyani K, Haghollahi F, Shahbazi Sighaldeh S. Sexual health definition from the perspective of Iranian experts and description its components. Tehran University Medical Journal. 2015;73(3):210-220.
21. Mohammed BH, Johnston JM, Harwell JI, Yi H, Tsang KW, Haidar JA. Intimate partner violence and utilization of maternal health care services in Addis Ababa, Ethiopia. BMC Health Serv Res. 2017;17(1):178. doi:10.1186/s12913017-2121-7

22. Rahman M, Nakamura K, Seino K, Kizuki M. Intimate partner violence and use of reproductive health services among married women: evidence from a national Bangladeshi sample. BMC Public Health. 2012;12:913. doi:10.1186/1471-2458-12-913

23. Reese Masterson A, Usta J, Gupta J, Ettinger AS. Assessment of reproductive health and violence against women among displaced Syrians in Lebanon. BMC Womens Health. 2014;14(1):25. doi:10.1186/1472-6874-14-25

24. Dolatian M, Hesami k, Shams J, Alavi Majd H, Moafi F. The barriers to screening for domestic violence. Iranian Journal of Forensic Medicine. 2012;17(4):225-232.

25. Bacchus L, Mezey G, Bewley S. Experiences of seeking help from health professionals in a sample of women who experienced domestic violence. Health Soc Care Community. 2003;11(1):10-18. doi:10.1046/j.13652524.2003.00402.x

26. Yargawa J, Leonardi-Bee J. Male involvement and maternal health outcomes: systematic review and meta-analysisJ Epidemiol Community Health. 2015;69(6):604-612. doi:10.1136/jech-2014-204784

27. Mullany BC. Barriers to and attitudes towards promoting husbands' involvement in maternal health in Katmandu, Nepal. Soc Sci Med. 2006;62(11):2798-2809. doi:10.1016/j. socscimed.2005.11.013

28. Kululanga LI, Sundby J, Chirwa E, Malata A, Maluwa A. Barriers to husbands' involvement in maternal health care in a rural setting in Malawi: a qualitative study. Journal of Research in Nursing and Midwifery. 2012;1(1):1-10.

(c) 2019 The Author (s); This is an open-access article distributed under the terms of the Creative Commons Attribution License (http://creativecommons.org/licenses/by/4.0), which permits unrestricted use, distribution, and reproduction in any medium, provided the original work is properly cited. 